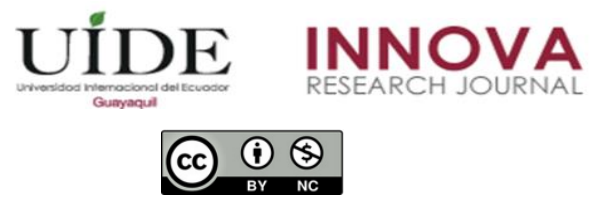

INNOVA Research Journal, ISSN 2477-9024

(Mayo-Agosto 2021). Vol. 6, No.2 pp. 36-61

DOI: https://doi.org/10.33890/innova.v6.n2.2021.1672

URL: http://revistas.uide.edu.ec/index.php/innova/index

Correo: innova@uide.edu.ec

\title{
Influencia de las estrategias heurísticas en el aprendizaje de la matemática
}

\section{Influence of heuristic strategies on learning mathematics}

Víctor Hugo Medina Pérez

Universidad César Vallejo, Perú

victormedina64@outlook.com

(D) https://orcid.org/0000-0002-0693-3827

Manuel Ángel Pérez Azahuanche

Universidad César Vallejo, Perú

manuelangelperez@gmail.com

https://orcid.org/0000-0003-4829-6544

Recepción: 02/01/2021 | Aceptación: 01/04/2021 | Publicación: 10/05/2021

Cómo citar (APA, séptima edición):

Medina Pérez, V. H., y Pérez Azahuanche, M. A. (2021). Influencia de las estrategias heurísticas en el aprendizaje de la matemática. Innova Research Journal, 6(2), 36-61. https://doi.org/10.33890/innova.v6.n2.2021.1672

\section{Resumen}

El estudio tuvo como finalidad determinar que las Estrategias heurísticas influyen en el Aprendizaje de la matemática en estudiantes de educación secundaria. Presenta un enfoque cuantitativo y diseño correlacional causal. Se desarrolló en 85 estudiantes a quienes se les aplicó un cuestionario y una prueba objetiva para identificar los niveles de ambas variables. Se basó en la esquematización y acciones de los estudiantes para investigar, organizar, sistematizar y analizar información, y comprender e interpretar el mundo que los rodea, usando métodos para resolver diferentes situaciones. Entre los hallazgos se obtuvieron un Rho Spearman de 0,915** que refiere a una correlación positiva muy alta y un Tau-b Kendall alta de 0,847. Se concluye que las Estrategias heurísticas facilitan la búsqueda de solución de problemas en el Aprendizaje de la matemática, conducen a decisiones precisas, con métodos que favorece a la reflexión. Finalmente, este artículo contribuye en el aspecto científico al conocer que mediante las Estrategias heurísticas promueven al Aprendizaje de la matemática.

Palabras claves: estrategias heurísticas; aprendizaje de la matemática; resolución de problemas. 


\begin{abstract}
The study aimed to determine that the Heuristic Strategies influence the learning of mathematics in high school students. It presents a quantitative approach and causal correlation design. It was developed in 85 students to whom a questionnaire and an objective test were applied to identify the levels of both variables. It was based on the student's schematization and actions to investigate, organize, systematize and analyze information, and understand and interpret the world around them, using methods to solve different situations. Among the findings were a Rho Spearman of $0.915^{* *}$ that refers to a very high positive correlation and a high Tau-b Kendall of 0.847 . It is concluded that the Heuristic Strategies facilitate the search of solution of problems in the Learning of Mathematics, they lead to precise decisions, with methods that favor the reflection. Finally, this article contributes in the scientific aspect when knowing that through the Heuristic Strategies they promote the Learning of Mathematics.
\end{abstract}

Keywords: heuristic strategies; mathematics learning; problem solving.

\title{
Introducción
}

El ser humano se encuentra en diversos contextos y tiene intercambio cultural y conocimiento matemático que lo lleva a usar estrategias y técnicas, para poder ejecutar las diversas actividades en las que se enfrenta, por ello es necesario, la comprensión en el área de matemática por los estudiantes, no enfocar en ellos la utilización de estrategias complejas o de instruirlos en cálculos complejos y considerando los software o programas tecnológicos que les permitan obtener resultados deseados. Para el logro de un aprendizaje en el estudiante es necesario desarrollar estrategias heurísticas durante el proceso de enseñanza, y así lograr las competencias matemáticas, asumiendo el enfoque del área de matemática referente a la solvencia de problemáticas y dar una salvedad crítica y coherente, con sustento de conocimientos lógicos y vinculados, de acuerdo a su realidad misma y en los contextos en los cuales se encuentren.

Los resultados internacionales que realiza el Programa para la Evaluación Internacional de Estudiantes (PISA) implementado por la Organización para la Cooperación y Desarrollo Económico (OCDE) quien evalúa a los estudiantes según las competencias que se logran en el área de matemática en escolares de 15 años, los cuales se encuentren en el último grado de la educación básica, es considerada como la evaluación internacional en lo que respecta a educación la cual es ejecutada cada tres años participando los de la OCDE países miembros. Participando Perú de manera facultativa. En el 2015 en Latinoamérica la proporción de escolares que lograron el nivel dos y los primeros lugares en la Región los puntajes de estos varían entre 50,7\% (Chile) y 9,4\% (República Dominicana). También el 33,9\% de estudiantes de Perú estuvieron en los niveles señalados los cuales tienen dificultades en la apropiada aplicación de Estrategias heurísticas para resolver problemas matemáticos. (Ministerio de Educación, 2017, p. 74).

Según el informe presentado por PISA en el 2018, el Perú tiene el 54\% de los estudiantes evaluados que se sitúan a partir del nivel 2 de desarrollo, como línea base para el dominio de la competencia financiera. Estos resultados son similares a los del 2015 y revelan que, de acuerdo a ello hay un gran desafío de seguir desarrollado esta competencia en las escuelas del país. Observándose que es necesario fortalecer las competencias matemáticas en todo el territorio del país. (Ministerio de Educación, 2018, p. 48). 


\section{Marco Teórico}

Las teorías que fundamentan el estudio están consideradas como las de; Vygotsky (1978), quien refiere que los problemas con los cuales se encuentran en relación los niños y jóvenes que aprenden, estos responden a las teorías del desarrollo los cuales están en relación con los procesos educativos, un poco confuso la relación existente entre el aprendizaje y el desarrollo del estudiante, todas las concepciones se centran en tres posiciones importantes. La primera, el proceso de desarrollo del estudiante independiente de su aprendizaje, en el cual este último se centra en el aspecto externo del individuo observándose en los logros de aprendizaje, la segunda posición el aprendizaje es desarrollo, basada en el concepto del reflejo, en el cual el aprendizaje del niño está unido al proceso de desarrollo. La tercera posición teórica, relación entre aprendizaje y desarrollo, anulando los externos, considerando la maduración del individuo el cual depende del sistema nervioso y por otro lado se encuentra el aprendizaje el cual también es un proceso que va evolucionado.

En el presente estudio se da a conocer las definiciones sobre las Estrategias heurísticas favorables en la educación de la formación de los estudiantes el Ministerio de Educación (2013, p. 123), manifiesta la instrucción es significativa, siendo el aprender un proceso objetivo y crucial: las personas que quieran aprender aprenderán. Además, se sabe que no hay forma o estrategia para dejar que una persona aprenda si es que no tiene el deseo de aprender. Es necesario un aprendizaje participativo, es preferible un proceso de aprendizaje aceptable, analítico y específico. El aprendizaje incluye la indagación y conocimiento "know how" habilidad o estrategia de solucionar problemas, crear presentaciones y revisar estrictamente las soluciones y presentaciones.

Cunaprioaru (2015), en todas las asignaturas escolares, la matemática propone y desarrolla problemas, soluciones y conceptos que son los componentes básicos en el campo. En matemáticas, la resolución de problemas es el concepto más eficaz, que se puede utilizar para contextualizar y recontextualizar conceptos, operaciones matemáticas básicas y transferencia dinámica de conocimientos para garantizar un aprendizaje sostenible y significativo. Hoon, et al. (2013) dan a conocer que el corazón para resolver problemas matemáticos es explorar y establecer la relación entre las diferentes ramas del conocimiento en matemáticas. El método heurístico puede explicar cómo estas ramas del conocimiento están conectadas a través de algunos principios generales básicos en el proceso de resolución de problemas matemáticos y transferencia de conocimiento matemático. Pouyamanesh y Firoozeh (2013), ellos expresan que la cantidad de matemáticas enseñada en la mayoría de los estudiantes que no toleran la frustración, y la tolerancia para los estudiantes que fallan en matemáticas es mejor la utilización de estrategias para obtener mejores resultados respecto a la comprensión de la matemática en las personas, por ello, el estudiante debe planificar, tener en cuenta procedimientos, la diagramación para emplear en lo posible y llegar a resultados favorables.

En cuanto a la conceptualización del Aprendizaje de la matemática, refiere MINEDU (2016, p. 235), que; aprender la matemática es una contribución a la formación de los ciudadanos, Pueden buscar, organizar, sistematizar y analizar información para comprender e interpretar el mundo que les rodea, juega un papel en él, tomar decisiones relevantes y utilizar los diversos métodos para resolver diferentes situaciones problemáticas: utilizando con flexibilidad pertinente 
métodos, estrategias y conocimientos matemáticos. Pólya (1965, p. 96), menciona que, cuando se trata de resolución de problemas, se basa en una perspectiva global, no solo en una perspectiva matemática. Su método de resolución de problemas se basa en una serie de procedimientos que serán manejados y realizados en todos los ámbitos de la vida diaria. La expresión es la siguiente: Desde tu punto de vista, lo más importante es que la idea de desarrollo matemático es la forma correcta de resolver problemas. Tenemos problemas en la vida cotidiana, en la ciencia y la política, en todas partes. Prasad, et al. (2020, p. 01) en el artículo, Incorporating STEAM Pedagogy in Teaching Mathematics, consideran a la matemática como un tema importante, como una fuente de creatividad e importante para los humanos, con el tiempo, la enseñanza y el aprendizaje de las matemáticas a nivel escolar se limita a la transferencia, siempre han considerado las matemáticas como una biblioteca predefinida de conocimientos y habilidades. Habiendo razones como la falta de conocimiento entre profesores y educadores, se debe tener en cuenta habilidades en el uso de métodos de enseñanza innovadores, en el proceso, parece que la motivación de los estudiantes para aprender matemáticas ha aumentado y siendo importante la participación de los estudiantes en matemáticas.

Muchos investigadores toman en cuenta la matemática para la formación en valores como Lissabet (2019) tuvo como eje central la ejecución del análisis y reflexión ante la resolución de problemas matemáticos y lograr en los estudiantes la formación de cualidades de la personalidad de los estudiantes específicamente influenciando en la esfera socio-moral. Según Arteaga y Macías, (2019), quien menciona que el problema incluye todas las situaciones que deben resolverse. Es necesario reflexionar, buscar información, razonar y usar estrategias, como piezas de un engranaje para revertir el mecanismo, lo que nos lleva así a la solución de los problemas. Esta no es una solución inmediata, sino muy bien analizada y meditada. Ante lo descrito se planteó la pregunta de investigación: ¿Cuál es la influencia de las Estrategias heurísticas en el Aprendizaje de la matemática en estudiantes de educación secundaria?, y como objetivo determinar la influencia de las Estrategias heurísticas en el Aprendizaje de la matemática y en cada una de sus dimensiones.

La habilidad resuelve problemas de forma, movimiento y posición, es decir, los estudiantes pueden orientarse y describir la posición y el espacio de los objetos y el movimiento en el espacio, visualizar con formas geométricas bidimensionales, explicar y relacionarse con la tridimensionalidad de lo específico y el objeto. Relacionado con la medición directa o indirecta de la superficie, el perímetro, el volumen y la capacidad del objeto, utiliza herramientas, estrategias y diseños para construir representaciones de formas geométricas para diseñar objetos, planos y modelos en cuanto a su diseño y medición. También se utilizan sistemas de referencia y lenguajes geométricos para describir caminos y rutas. Y finalmente resuelve problemas de la gestión de datos y la incertidumbre, el alumno puede analizar la información sobre el tema de utilidad, aprendizaje o situaciones aleatorias, de manera que pueda tomar decisiones, hacer predicciones y conclusiones razonables y obtener los resultados de soporte de información. Para ello, los estudiantes recopilan, organizan y representan datos utilizando estadísticas y medidas de probabilidad para proporcionar datos para el análisis, interpretación y conclusión del proceso determinista o aleatorio en la situación.

Las teorías que han fundamentan el estudio del aprendizaje de la matemática tenemos a lo respaldado filosóficamente por la Teoría de situaciones didácticas formulada por Brousseau, G. 
(1999), menciona que el sujeto aprende adaptándose a un medio resistente con el que interactúa. Su saber es fruto de la adaptación, dando respuestas nuevas lo cual es prueba de su aprendizaje. También la Teoría de las inteligencias de múltiples Gardner (1995), mencionando a: Inteligencia lógico matemática capacidad de razonar, calcular, tener un pensamiento lógico y organizar los objetos de manera sistemática y la Inteligencia visual espacial, para poder comprender y expresar imágenes visuales y espaciales en el desarrollo de problemas. Asimismo, se toma en cuenta a la Teoría de Resolución de Problemas, formulada por Schoenfeld (1985), en el que se debe tener en cuenta diversas situaciones como: los recursos, control, sistema de creencias. El enfoque cada vez más influye en las matemáticas en el procedimiento de resolver problemas, que tenga las características de: ser un producto cultural dinámico que se desarrolla y adapta constantemente. Todas las actividades matemáticas en su fase pueden resolver el problema causado por situaciones, los eventos importantes se consideran que ocurren en diferentes situaciones. La situación se divide en cuatro categorías: situaciones cuantitativas; situaciones de regularidad, de reciprocidad y cambio; de forma, manejo y ubicación y de datos e incertidumbre. En la resolución de los problemas del área de matemática es necesario una serie de pasos, diseños, exploración y elaboración de formas de visualizar alternativas de solución para ello Schoenfeld (1985), propuso un modelo para resolver problemas que consistía de las siguientes etapas: el análisis orientado a la comprensión del problema mediante la construcción de una representación adecuada. Diseño de un plan de solución global. Exploración orientada a la transformación del problema en una tarea rutinaria. Elaborar un plan de solución. Verificar la solución.

La teoría de Ausubel (1961, p. 64), describe que el aprendizaje en el estudiante estriba de su estructura cognitiva con relación de lo presente con el conocimiento, con las ideas que la persona posee en un determinado aspecto, siendo necesario el conocer su estructura cognitiva. Por lo que es inevitable tener en cuenta que tipo de conceptos maneja y también el nivel de estabilidad emocional del estudiante. Además, el lenguaje es una parte importante del aprendizaje significativo el cual se basa en la recepción y también en el descubrimiento, cada vez que el individuo manipula conceptos y proposiciones refina su comprensión verbal y surge el aprendizaje significativo lo cual hace que sean más precisos y transferibles. Esto permite que el estudiante a medida que desarrolla, su concepto va definiendo, lo cual le permite realizar un análisis y síntesis de lo aprendido en matemática.

Tejeda (2017), da a conocer las clases de Estrategias heurísticas utilizables como el tanteo y error, lo que implica una selección aleatoria de soluciones o medidas y la aplicación de las condiciones del problema a estos resultados o medidas hasta encontrar o validar una meta. Después de la primera prueba, las alternativas ya no se seleccionan al azar, sino que se tienen en cuenta las pruebas que se han realizado. La estrategia Resuelva un problema similar y más simple, permite obtener una solución, a menudo es útil resolver primero el mismo problema con datos más simples y luego usar el mismo método para resolver problemas más complejos, usando gráficos, tablas, en los problemas, se puede encontrar fácilmente una solución si crea un gráfico o diagrama. Wang (2012), también hace mención que el pensamiento matemático se desarrolla al moldear y desarrollar los hábitos necesarios para resolver problemas. Por ello, el mejor recurso para estimular el desarrollo de ideas es preguntándose cómo resolver problemas, siendo importante siempre tener en cuenta todos los aspectos del pensamiento matemático y empelarlos en todas las fases de sus actividades. Cuba (2015) refiere que para buscar o encontrar una solución, y no conocemos el 
algoritmo de la solución, se utilice procedimientos heurísticos, principios heurísticos, reglas, estrategias y procedimiento, considerados como heurísticas los diagramas de análisis, esquemas, diagramas explicativos, tablas, gráficos, resúmenes de fórmulas, entre otros. El principio heurístico es muy útil para encontrar nuevos conocimientos y también puede proporcionar sugerencias para resolver varios problemas.

Tambunan (2018), hace mención que los resultados muestran que las Estrategias heurísticas tienen una gran influencia en la capacidad matemática de los estudiantes en el pensamiento de orden superior, mientras que algunas estrategias didácticas pueden afectar las habilidades de los estudiantes en comprensión de conceptos, creatividad, comunicación matemática, resolución de problemas y capacidad de razonamiento. Novotná, et al. (2014), quienes utilizan las Estrategias heurísticas para resolver problemas como: analogía, supuestos-verificación, experimentos del sistema, reformulación del problema, diseño de soluciones, regresión y uso de diagramas de funciones. Eisenmann, et al. (2015), manifiestan resultados importantes, donde la identificación de métodos heurísticos y la determinación de estrategias no heurísticas pueden adoptarse para soluciones a largo plazo en los problemas apropiados. El contenido que se puede enseñar incluye experimentación sistemática, adivinación, verificación, revisión e introducción de herramientas de ayuda.

Monereo (1998, p. 48), precisa que Estrategias heurísticas son las que se tiene que asumir y que no existe por separado sino en conjunto, en la resolución de dificultades, las cuales dirigen un camino, una forma o guía en la absolución de los problemas planteados de manera favorable positiva ante estos trazados. Polya (1997, p. 95), enfatiza que la heurística se enfoca en encontrar y buscar soluciones y disfrutar de la experiencia de cómo lo hacen los estudiantes, de modo que haya búsqueda creativa o basada en la experiencia e investigación racional. Peralta (2000, p.64), da a conocer que, las Estrategias heurísticas son actividades psicológicas de los estudiantes en el proceso de aprendizaje, donde el estudiante es el sujeto principal y el centro de aprendizaje, y la tarea del docente es despertar el interés, incentivar y orientar el aprendizaje de los alumnos. En el proceso de resolución de problemas, el maestro lo seguirá para ayudar a resolver errores y convertirlos en una oportunidad de aprendizaje.

Boscán y Klever (2012, p. 04), reconocen como dimensiones de Estrategias heurísticas a: Comprensión del enunciado, identificar los datos de los problemas, identificar si los existentes son suficientes para ejecutar el proceso, reconocer la incógnita en el problema a resolver, identificar si los datos existentes son suficientes para ejecutar el proceso. Concepción de un plan, buscar estrategias que ayuden a la solución del problema, herramientas que ayuden a resolver cada problema planteado, elaborar gráficos para resolver, explicar el plan pertinente que ayuda a solucionar el problema. Ejecución del plan, aplicar cada una de las estrategias que ha hecho, utilizar alguna estructura matemática para la solución del problema y utilizar operaciones simbólicas que ayuden a la resolución de los problemas. Visión retrospectiva, se comprueban y comparan la solución al problema con otros que le sirvan de apoyo. Repiensan, evalúan la solución a los problemas, y cambian los datos del problema y contextos propuestos para resolver problemas.

Aggarwal y Bal (2020) en el artículo titulado; Tools of ict for learning and teaching mathematics, mencionan que es también necesario la utilización de programas que ofrecen las tecnologías de la comunicación y de la información, las cuales permiten el desarrollo de 
habilidades de orden superior aumentando así el interés de los estudiantes en el área de matemática. Al educar a los escolares para que, se conviertan en estudiantes de por vida y en empleados exitosos en el lugar de trabajo y en la sociedad, se deben reemplazar los métodos tradicionales de enseñanza y aprendizaje. Shakirova, et al. (2018) hacen referencia que, la motivación es uno de los principales requisitos previos para la mejora de la calidad de la enseñanza de las matemáticas. La motivación en el estudio, depende de una serie de factores: el sistema educativo, la organización del proceso educativo, las características individuales del educador y del estudiante, la motivación en la enseñanza de las matemáticas. La investigación y la experiencia pedagógica indican que, tanto los estudiantes como los profesores que están empezando su carrera subestiman el papel de la motivación en la enseñanza de las matemáticas, y sus habilidades de la motivación son insuficientes, siendo necesario un replanteamiento para subsanar en parte fundamental en el emprendimiento de su profesión.

Mani, et al. (2020), hacen referencia que, en varias investigaciones se da a conocer que, enseñar matemáticas ha sido una tarea difícil para muchos maestros, la mayoría de los profesores de matemáticas, se encuentran luchando por mejorar sus habilidades pedagógicas para proporcionar a los estudiantes un aprendizaje significativo (auténtico, empoderado, justificable e inclusivo). Revelo (2018), también menciona que, el papel de la tecnología e internet en el aprendizaje de las matemáticas puede generar cierta motivación, pero estos no representan que los estudiantes y los profesores tengan un impacto significativo o alto en el aprendizaje de las matemáticas a largo plazo. No es por su uso o disponibilidad, sino porque carecen de capacidad para aplicarles en sus estudios. Sáenz, et al. (2017), aluden que al evaluar la efectividad del método heurístico de Polya (1981), en el desarrollo de la habilidad matemática en el pensamiento espacial, en estudiantes de quinto grado, al finalizar sus estudios de nivel básico ellos si consideraron los pasos de resolución de problemas del modelo Polya y la estrategia de colaboración. Luego de la utilización, su desempeño mejoró significativamente, confirmando la efectividad de la estrategia, dando una mayor claridad la aplicación del método de Poyla para desarrollar problemas matemáticos. Hitt y Quiroz (2018), refieren que las intuiciones de los estudiantes son funcionales y espontáneas y pueden modificarse mediante la interacción y el diálogo en el trabajo en equipo. Esto promueve la ecuanimidad en la construcción del conocimiento. Para la construcción de contenidos es necesario tener en cuenta la relación de los estudiantes el intercambio de ideas para la formación de un nuevo tema, el trabajo colaborativo permite la construcción de nuevas formas de pensar.

Para el aprendizaje del escolar en la educación básica regular en el área de matemática es necesario lograr competencias del área, consideradas en este estudio como dimensiones del Aprendizaje de la Matemática: La solución de problemas de cantidad, donde el estudiante enmienda problemas o plantea unos nuevos, los cuales accedan a edificar y alcanzar las nociones de cantidad de número, de sistemas numéricos, sus operaciones y propiedades. Vale la pena enfatizar en el razonamiento lógico que usan los estudiantes al hacer comparaciones. Resolución de problemas de regularidad, equivalencia y variación, que se basan en la capacidad de descubrir valores desconocidos, establecer límites y hacer predicciones sobre el comportamiento del fenómeno. Resolver problemas de forma, movimiento y posición, es decir, los estudiantes pueden orientarse y describir la posición y el espacio de los objetos y el movimiento en el espacio, visualizar con formas geométricas bidimensionales, explicar y relacionarse con la 
tridimensionalidad de lo específico y el objeto. Y solucionar problemas de gestión de datos y de incertidumbre, en donde se puede analizar la información sobre el tema de utilidad, realizar un aprendizaje a situaciones aleatorias, de manera que pueda tomar decisiones, hacer predicciones, llegar a conclusiones razonables y obtener los resultados. (Ministerio de educación, 2016).

Arias, et al. (2018), en; Estrategias heurísticas en resolución de problemas a través de una experiencia integradora, publicado en la Revista Sarance No 42, se basó en el modelo cualitativo y cuantitativo. Aplicaron el trabajo colaborativo. Concluyeron que es importante planificar, ejecutar las experiencias integradoras en el aula para la reflexión sobre el aprendizaje que están ejecutando, promoviendo aprendizajes con autonomía y colaborativos. El trabajo de Neyra (2020), titulado; Aprendizaje Basado en Problemas para el Aprendizaje significativo en Matemática, en estudiantes de tercer año de secundaria, Chao 2019. Desarrolló la investigación cuantitativa con diseño cuasi experimental. Con U de Mann-Whitney, determinó que al aplicar estrategias del Aprendizaje Basado en Problemas (ABP) da un efecto positivo en mejora del aprendizaje significativo de matemática con un p-valor de 5\% y el nivel de logrando el $45 \%$ en proceso después de la aplicación de la estrategia. Asimismo, en el estudio de Tejeda (2017), en el estudio; Estrategias heurísticas y clima escolar en el aprendizaje de la matemática en estudiantes de primer año de secundaria de la Red 2 de la UGEL 03-2015, que el 47,8\% tiene tendencia moderada en relación a la aplicación de estrategias heurísticas, el nivel de logro alcanzado fue de 77,3\% en el aprendizaje de la matemática. Concluyendo la existencia positiva y alta de correlación entre las variables.

\section{Metodología}

De acuerdo al enfoque que persigue es cuantitativo, de tipo no experimental y explicativo. Según Hernández et al, (2010) los datos son utilizados para la contrastación de la hipótesis, los cuales son de medición numérica, regidos de acuerdo a las teorías. Presenta un diseño correlacional causal, de corte transversal, debido a la finalidad que persiguen las variables, en donde se observa si la primera o independiente influye en la segunda o dependiente en un contexto en particular en el cual se ejecutó el estudio.

Se aplicó a 85 estudiantes del quinto grado de educación secundaria. Para la primera variable un cuestionario, cuyo conjunto de preguntas sistematizadas estuvieron relacionadas con los indicadores de la variable en estudio, con 16 ítems y su escala tipo Likert con criterios siempre (4), casi siempre (3), A veces (2) Nunca (1), distribuidas las preguntas en comprensión del enunciado, (4 ítems) concepción de un plan (4 ítems), ejecución del plan (4 ítems), visión retrospectiva (4 ítems). Respecto a la segunda variable en estudio se utilizó la prueba objetiva, constituida por 16 preguntas, con alternativas de tipo complemento único distribuidas en las dimensiones descritas anteriormente referente a la variable en estudio, cada una de ellas estuvo constituida por cuatro problemas, facilitando el recojo pertinente de los datos. Ambos instrumentos fueron sometidos a procesos de validación y confiabilidad.

Los instrumentos han sido elaborados por los autores y validados por 6 expertos. (Anexos) 


\section{Resultados}

Se presentan los resultados obtenidos referentes a las variables Estrategias heurísticas y Aprendizaje de la matemática, recolectados por los instrumentos, datos alcanzados por los estudiantes del quinto grado de educación secundaria.

\section{Tabla 1}

Prueba de normalidad para las variables Estrategias heurísticas y Aprendizaje de la matemática

\begin{tabular}{lccc}
\hline & \multicolumn{3}{c}{ Kolmogorov-Smirnov $^{\mathbf{a}}$} \\
\cline { 2 - 4 } & Estadístico & $\mathrm{gl}$ & Sig. \\
\cline { 2 - 4 } Estrategias heurísticas & 0.102 & 85 & 0.030 \\
Aprendizaje de matemática & 0.150 & 85 & 0.000 \\
\hline
\end{tabular}

Nota. Según datos obtenidos de la matriz.

Al realizar la prueba de contraste de la normalidad mediante Kolmogorov-Smirnov, se observa que, para Estrategias heurísticas y Aprendizaje de la matemática el grado de significancia para ambas variables es menor a 0,05 , por lo cual se asume que los datos no tienen una distribución normal.

\section{Tabla 2}

Frecuencia de estudiantes por niveles y variables

\begin{tabular}{ccccc}
\hline Variables & \multicolumn{2}{c}{ Estrategias heurísticas } & \multicolumn{2}{c}{ Aprendizaje de la matemática } \\
\cline { 2 - 5 } Niveles & $\mathrm{f}$ & $\%$ & $\mathrm{f}$ & $\%$ \\
\hline Logro destacado & 22 & 26 & 54 & 27 \\
Logro esperado & 53 & 62 & 8 & 64 \\
Proceso & 10 & 12 & 0 & 0 \\
Inicio & 0 & 0 & 85 & 100 \\
\hline Total & 85 & 100 & & 0 \\
\hline
\end{tabular}

Nota. Datos obtenidos de la matriz; $\mathrm{f}=$ Frecuencia absoluta; $\%=$ Frecuencia relativa.

Los estudiantes se encuentran en su mayoría en logro destacado y esperado $88 \%$ para la primera variable y $91 \%$ para la segunda variable y una minoría en proceso $12 \%$ y $9 \%$ respectivamente. 


\section{Tabla 3}

Relación entre las variables Estrategias heurísticas y Aprendizaje de la matemática

\begin{tabular}{|c|c|c|c|c|}
\hline \multicolumn{5}{|c|}{ Correlaciones } \\
\hline & & & $\begin{array}{l}\text { Estrategias } \\
\text { heurísticas }\end{array}$ & $\begin{array}{l}\text { Aprendizaje de la } \\
\text { matemática }\end{array}$ \\
\hline \multirow[t]{6}{*}{ Rho de Spearman } & \multirow[t]{3}{*}{$\begin{array}{l}\text { Estrategias } \\
\text { heurísticas }\end{array}$} & $\begin{array}{l}\text { Coeficiente de } \\
\text { correlación }\end{array}$ & 1,000 &, $915^{* *}$ \\
\hline & & Sig. (bilateral) & . &, 000 \\
\hline & & $\mathrm{N}$ & 85 & 85 \\
\hline & \multirow{3}{*}{$\begin{array}{l}\text { Aprendizaje } \\
\text { de la } \\
\text { matemática }\end{array}$} & $\begin{array}{l}\text { Coeficiente de } \\
\text { correlación }\end{array}$ & $915^{* *}$ & 1,000 \\
\hline & & Sig. (bilateral) & ,000 & \\
\hline & & $\mathrm{N}$ & 85 & 85 \\
\hline
\end{tabular}

**. La correlación es significativa en el nivel 0,01 (bilateral). Tau-b Kendall =00.847 sig. $\mathrm{P}<0.01$

Nota. Datos obtenidos de la matriz; $\mathrm{N}=$ Muestra.

Se muestra en la Tabla 3, la relación que existe entre las variables en estudio que según Rho Spearman es de $0,915^{* *}$, correlación positiva muy alta y muy significativa; con p-valor menor a 0,05 entre las Estrategias heurísticas y el Aprendizaje de la matemática y según Tau-b Kendall es alta de 0,847 , lo cual determina que existe influencia de la variable independiente sobre la dependiente.

\section{Tabla 4}

Relación entre las Estrategias heurísticas y Resuelve problemas de cantidad del Aprendizaje de la matemática

\begin{tabular}{|c|c|c|c|c|}
\hline \multicolumn{5}{|c|}{ Correlaciones } \\
\hline & & & $\begin{array}{r}\text { Estrategia } \\
\text { s heurísticas }\end{array}$ & $\begin{array}{l}\text { Resuelve problemas de } \\
\text { cantidad }\end{array}$ \\
\hline \multirow[t]{6}{*}{ Rho de Spearman } & \multirow{3}{*}{$\begin{array}{l}\text { Estrategias } \\
\text { heurísticas }\end{array}$} & Coeficiente de correlación & 1,000 &, $658^{* * *}$ \\
\hline & & Sig. (bilateral) & . &, 000 \\
\hline & & $\mathrm{N}$ & 85 & 85 \\
\hline & \multirow{3}{*}{\begin{tabular}{l}
\multicolumn{1}{c}{ Resuelve } \\
problemas de \\
cantidad
\end{tabular}} & Coeficiente de correlación &, $658^{* *}$ & 1,000 \\
\hline & & Sig. (bilateral) &, 000 & \\
\hline & & $\mathrm{N}$ & 85 & 85 \\
\hline
\end{tabular}

**. La correlación es significativa en el nivel 0,01 (bilateral). Tau-b- Kendall $=0.556$ sig. $\mathrm{p}<0.05$

Nota. Datos obtenidos de la matriz; N= Muestra.

En la Tabla 4, al relacionar los datos en cuanto a la variable Estrategias heurísticas y Resuelve problemas de cantidad del Aprendizaje de la matemática, según Rho Spearman el valor que se obtiene es $0,658^{* *}$, positiva alta y muy significativa y según Tau-b Kendall el valor obtenido es de 0,556 media, quedando demostrada la influencia, es decir al aumentar las Estrategias heurísticas aumenta la Resolución de problemas de cantidad y un mejor Aprendizaje de la matemática en los estudiantes del quinto año de educación secundaria. 


\section{Tabla 5}

Relación entre las Estrategias heurísticas y Resuelve problemas de regularidad, equivalencia y cambio del Aprendizaje de la matemática

\begin{tabular}{|c|c|c|c|c|}
\hline \multicolumn{5}{|c|}{ Correlaciones } \\
\hline & & & $\begin{array}{l}\text { Estrategias } \\
\text { heurísticas }\end{array}$ & $\begin{array}{l}\text { Resuelve problemas de } \\
\text { regularidad, equivalencia y cambio }\end{array}$ \\
\hline \multirow[t]{6}{*}{ Rho de Spearman } & \multirow[t]{3}{*}{$\begin{array}{l}\text { Estrategias } \\
\text { heurísticas }\end{array}$} & $\begin{array}{l}\text { Coeficiente de } \\
\text { correlación }\end{array}$ & 1,000 &, $477^{* *}$ \\
\hline & & Sig. (bilateral) & . & ,000 \\
\hline & & $\mathrm{N}$ & 85 & 85 \\
\hline & \multirow{3}{*}{$\begin{array}{l}\text { Resuelve } \\
\text { problemas de } \\
\text { regularidad, } \\
\text { equivalencia y } \\
\text { cambio }\end{array}$} & $\begin{array}{l}\text { Coeficiente de } \\
\text { correlación }\end{array}$ & $477^{* *}$ & 1,000 \\
\hline & & Sig. (bilateral) &, 000 & . \\
\hline & & $\mathrm{N}$ & 85 & 85 \\
\hline
\end{tabular}

**. La correlación es significativa en el nivel 0,01 (bilateral). Tau-b- Kendall = 0.453 sig. $\mathrm{p}<0.05$

Nota. Datos obtenidos de la matriz; N= Muestra

En la Tabla 5, al ejecutar la relación de los datos según Rho Spearman, se obtuvo el valor de $0,477 * *$ positiva moderada y muy significativa y según Tau-b Kendall el valor obtenido es 0,453 es media lo que demuestra la influencia, es decir, al aumentar las Estrategias heurísticas aumenta de manera moderada la Resolución de problemas de regularidad, equivalencia y cambio en el Aprendizaje de la matemática en los estudiantes de quinto año de educación secundaria.

\section{Tabla 6}

Relación entre las Estrategias heurísticas y Resuelve problemas de forma, movimiento y localización del Aprendizaje de la matemática

\begin{tabular}{|c|c|c|c|c|}
\hline \multicolumn{5}{|c|}{ Correlaciones } \\
\hline & & & $\begin{array}{r}\text { Estrategia } \\
\text { s heurísticas }\end{array}$ & $\begin{array}{l}\text { Resuelve problemas de forma, } \\
\text { movimiento y localización }\end{array}$ \\
\hline \multirow[t]{6}{*}{ Rho de Spearman } & \multirow[t]{3}{*}{$\begin{array}{l}\text { Estrategias } \\
\text { heurísticas }\end{array}$} & $\begin{array}{l}\text { Coeficiente } \mathrm{d} \\
\text { correlación }\end{array}$ & 1,000 &, $493^{* * *}$ \\
\hline & & Sig. (bilateral & . & ,000 \\
\hline & & $\mathrm{N}$ & 85 & 85 \\
\hline & \multirow{3}{*}{$\begin{array}{l}\text { Resuelve } \\
\text { problemas de } \\
\text { forma, } \\
\text { movimiento y } \\
\text { localización }\end{array}$} & $\begin{array}{l}\text { Coeficiente } \mathrm{d} \\
\text { correlación }\end{array}$ &, $493^{* *}$ & 1,000 \\
\hline & & Sig. (bilateral & ,000 & \\
\hline & & $\mathrm{N}$ & 85 & 85 \\
\hline
\end{tabular}

**. La correlación es significativa en el nivel 0,01 (bilateral). Tau-b- Kendall $=0.380$ sig. $\mathrm{p}<0.05$

Nota. Datos obtenidos de la matriz; N= Muestra 
En la Tabla 6, al realizar la relación de los datos según Rho Spearman es de 0,493** positiva moderada y muy significativa y según Tau-b Kendall es de 0,453 es media demostrando influencia, es decir, al aumentar las Estrategias heurísticas aumenta moderadamente la Resolución de problemas de forma, movimiento y localización y un mejor Aprendizaje de la matemática en los estudiantes.

\section{Tabla 7}

Relación entre las Estrategias heurísticas y Resuelve problemas de gestión de datos e incertidumbre del Aprendizaje de la matemática

\begin{tabular}{|c|c|c|c|c|}
\hline \multicolumn{5}{|c|}{ Correlaciones } \\
\hline & & & $\begin{array}{l}\text { Estrategias } \\
\text { heurísticas }\end{array}$ & $\begin{array}{l}\text { Resuelve problemas de } \\
\text { gestión de datos e } \\
\text { incertidumbre }\end{array}$ \\
\hline \multirow[t]{6}{*}{ Rho de Spearman } & \multirow{3}{*}{$\begin{array}{l}\text { Estrategias } \\
\text { heurísticas }\end{array}$} & Coeficiente de correlación & 1,000 &, $501^{* *}$ \\
\hline & & Sig. (bilateral) & . &, 000 \\
\hline & & $\mathrm{N}$ & 85 & 85 \\
\hline & \multirow{3}{*}{\begin{tabular}{l}
\multicolumn{1}{c}{ Resuelve } \\
problemas de \\
gestión de \\
datos e \\
incertidumbre
\end{tabular}} & Coeficiente de correlación &, $501^{* *}$ & 1,000 \\
\hline & & Sig. (bilateral) & ,000 & \\
\hline & & $\mathrm{N}$ & 85 & 85 \\
\hline
\end{tabular}

**. La correlación es significativa en el nivel 0,01 (bilateral). Tau-b- Kendall = 0.521 sig. p <0.05

Nota. Datos obtenidos de la matriz; N= Muestra

En la tabla 7, al realizar la relación de los datos según Rho Spearman es de 0,501** positiva moderada y muy significativa. Según Tau-b Kendall es 0.521 es media demostrando influencia; es decir, al aumentar la las Estrategias heurísticas aumenta de forma moderada la Resolución de problemas de gestión de datos e incertidumbre y un mejor Aprendizaje de la matemática en los estudiantes.

\section{Discusión}

En las estrategia heurísticas se observó que los estudiantes promueven estrategias que les facilita la búsqueda independiente de soluciones a los problemas propuestos, en un $62 \%$ en el nivel logro esperado; también se observó en la variable Aprendizaje de matemática que los estudiantes pueden investigar, organizar, sistematizar y analizar información y así comprender e interpretar la problemática del contexto, tomar decisiones relevantes y usar métodos para solucionar las problemas de manera estratégica, reflexiva y con conocimiento matemático observando que el 64\%, se encuentran en el nivel logro esperado, en los estudiantes. Coincidiendo con Arias, et al. (2018), que mencionan la importancia de planificar, ejecutar las experiencias integradoras, las que influirán en el aprendizaje, promoviendo la autonomía y el trabajo colaborativo con sus compañeros. Refuerza la conclusión de Medina (2017), quien manifiesta que, la competencia matemática referente a la resolución de problemas se utiliza en un contexto relacionado con la vida 
cotidiana del estudiante, por ello es de suma importancia poder tenerlo en cuenta para solucionar problemas que se relacionan con el contexto del ser humano.

La causa y efecto de las variables según Rho Spearman debido a que los datos son no normales, es de 0,915**, entre las Estrategias heurísticas y Aprendizaje de la matemática siendo positiva muy alta y muy significativa con un p-valor menor a 0,05 , con un grado de correlación según Tau-b Kendall es de 0.847. Por lo que si se pone énfasis en la utilización de las Estrategias heurísticas por lo tanto aumentará también el Aprendizaje de la matemática en los estudiantes. Sobre las Estrategias heurísticas y su incidencia en la Resolución de problemas de cantidad, la correlación encontrada según Rho Spearman es de $0,658^{* *}$, positiva alta y muy significativa según Tau-b Kendall de 0.556. Por lo tanto, se puede afirmar que si se aumenta la incidencia en utilizar. Para comparar con los resultados Solis (2019), por medio de Chi cuadrada encontrado de 97,7\% con significancia menor a 0,05 y concluye que las estrategas heurísticas influyen de manera significativa al aprender la matemática. Respecto a las Estrategias heurísticas en la Resolución de problemas de regularidad, equivalencia y cambio en el Aprendizaje de la matemática se obtuvo una correlación de $0,477 * *$ Rho Spearman, positiva moderada y muy significativa, según Tau-b Kendall es de 0.453, al igual que Tejeda (2017), quien manifiesta que referente al nivel de Aprendizaje de la matemática fue de $77,3 \%$ en nivel de logro, además señala que encontró una correlación positiva y alta entre las Estrategias heurísticas y clima escolar en el Aprendizaje de la matemática. Por su parte, Navarro y Deulofeu (2018) mencionan que el juego de estrategias influye en la solución a los problemas, el lenguaje que se utiliza en las soluciones por parte del estudiante, son muy favorables en todos los aspectos estudiados, verificando que, si aumentan la utilización de estrategias según el problema, entonces la solución será la más pertinente.

Las Estrategias heurísticas aumentará también la Resolución de problemas de forma, movimiento y localización en los estudiantes, se demuestra al obtener un Rho Spearman de $0,493^{* *}$, positivo moderado y muy significativo, y un Tau-b Kendall es de 0.453; al igual que Alarcón (2016), al señalar que la estrategia didáctica mejora significativamente la resolución de problemas en el área de matemática en el logro de sus aprendizajes en los estudiantes. Asimismo, Minotta (2015) refiere que es muy influyente las estrategias tácticas que, los escolares utilizan en la matemática, describiendo el paso a paso desde cómo se enfoca al problema, como se plantea las preguntas las cuales sean propicias y significativas al momento de resolver los problemas, dando realce al estudio propuesto en el que se puede ver que en diversos contextos se viene utilizando las estrategias como formas de dar solución a los problemas del entorno.

Se demuestra que al aumentar las Estrategias heurísticas aumenta también la Resolución de problemas de gestión de datos e incertidumbre según Rho Spearman de 0,501**, positiva moderada y muy significativa, según Tau-b Kendall es de 0.521. Las Estrategias heurísticas favorecen enormemente en la resolución de problemas de gestión de datos e incertidumbre para el Aprendizaje de la matemática; coincidiendo con Neyra (2020), quien menciona que el Aprendizaje de la matemática tiene su base en el aprendizaje basado en problemas, lo cual fundamenta la investigación en su estudio, encontró que disminuyó en un 54\% el nivel inicio, esto nos aclara aún más el incremento de utilizar problemas en mejora del aprendizaje de los estudiantes, los cuales buscan las tácticas más pertinentes y sistemáticas para solucionar los problemas en relación a su entono. 
De acuerdo a lo mencionando por el Ministerio de Educación (2016), las competencias logradas por el estudiante permiten enfrentar circunstancias problemáticas del entorno social, con el que se relacionan por motivos imprevisibles o de trabajo para enmendar problemáticas de su vida familiar o de su entorno. El Aprendizaje de la matemática implica la aplicación de diversas acciones que articulen de manera coordinada con la adecuación y aplicabilidad de estrategias y lograr las competencias del área de matemática, éstas se enmarcan en un contexto que permiten el desarrollo del estudiante en esa etapa de su vida y a futuro en otros contextos en los que se pueda encontrar, por tal motivo es favorable inculcar la utilización de estrategias en el área de matemática.

El aporte de la Teoría de Vygotsky (1925), para el desarrollo en el aprendizaje también está vinculado con su personalidad, donde plantea en primera instancia realizar una observación del logro de aprendizaje para luego poder ver cómo va desarrollándose, y en última instancia comparar el desarrollo de aprendizaje y el personal, en lo cual se puede apreciar que ambos van desarrollándose al mismo tiempo. Esta teoría permite reafirmarlo con la presente investigación debido a que el individuo a medida que va desarrollando este alcanza nuevos conocimientos, los cuales intervienen en la formación de ellos mismos mejorando su estatus y su relacionamiento con su entorno.

Asimismo, el aporte de la Teoría de Ausubel (1961) propone un mecanismo que se da en el estudiante al aprender algo nuevo lo relaciona con un concepto previo ya aprendido y que este genera un nuevo aprendizaje que cala en la parte cognitiva, observando una recepción de algo nuevo para construir ideas y conceptos, además existe el afianzamiento del lenguaje que a medida que va descubriendo algo nuevo va describiéndolo y enriqueciendo su concepción, y cada vez que manipula conceptos y proposiciones refina su comprensión verbal y surge el aprendizaje significativo haciendo que se vuelva preciso e intransferible propio de su mismo aprendizaje. Estas concepciones teóricas afirman lo que el alumno va aprendiendo a medida de su proceso educativo analizando y sintetizando de acuerdo a los conceptos matemáticos y en la absolución de problemas, en el cual también va formándose con valores de responsabilidad, honestidad y veracidad entre otros, siendo favorable en la medida que sigue desarrollándose.

Asimismo, Schoenfeld (1985), propone un modelo en la absolución de problemas por etapas: el análisis está orientado a la comprensión del problema a través de la construcción de una representación adecuada, también el diseño de un plan de solución global favorece la solución de problemas, la exploración orientada a la transformación del problema en una tarea rutinaria, también el elaborar un plan de solución y verificar la solución del problema; estas acciones son favorables para la resolución de problemas.

\section{Conclusiones}

Las Estrategias heurísticas influyen significativamente en el Aprendizaje de la matemática en estudiantes de educación secundaria, al obtener una correlación de $0,915^{* *}$, positiva muy alta y muy significativa según Rho Spearman, también según Tau-b Kendall la correlación es de 0.847 con un p-valor menor a 0,01, permitiendo deducir que al aumentar el uso de las Estrategias 
heurísticas aumenta también el Aprendizaje de la matemática en los estudiantes del quinto año de secundaria.

La influencia de las Estrategias heurísticas en la dimensión Resuelve problemas de cantidad es de $0,658^{* *}$, positiva alta y muy significativa según Rho Spearman con el p-valor menor a 0,05 y según Tau-b Kendall es de 0.556, al utilizar las Estrategias heurísticas aumentará la Resolución de problemas de cantidad en Aprendizaje de la matemática en los estudiantes del quinto año de secundaria.

La influencia de las Estrategias heurísticas en la dimensión Resuelve problemas de regularidad, equivalencia y cambio es de $0,477^{* *}$, positiva moderada pero muy significativa, según Rho Spearman con el p-valor menor a 0,05, según Tau-b Kendall es de 0.453, al usar las Estrategias heurísticas aumentará la absolución de problemas de regularidad, equivalencia y cambio y un mejor Aprendizaje de la matemática en estudiantes del quinto año de secundaria.

La influencia de las Estrategias heurísticas en la dimensión Resuelve problemas de forma, movimiento y localización es de $0,493^{* *}$, positiva moderada y a la vez muy significativa, según Rho Spearman p-valor menor a 0,05, al ejecutar las Estrategias heurísticas aumenta el desarrollo de los problemas de forma, movimiento y localización para un mejor Aprendizaje de la matemática en estudiantes del quinto año de secundaria.

La influencia de las Estrategias heurísticas en la dimensión Resuelve problemas de gestión de datos e incertidumbre en estudiantes de educación secundaria, Trujillo 2020, es de 0,501**, positiva moderada y a la vez es muy significativa, según Rho Spearman p-valor menor a 0,05, según Tau-b Kendall de 0.453, al emplear las Estrategias heurísticas aumenta la Resolución de problemas de gestión de datos e incertidumbre y un mejor Aprendizaje de la matemática en los estudiantes del quinto año de secundaria.

\section{Referencias Bibliográficas}

Aggarwal, Madhu \& Bal, Satinder. (2020). Tools of ICT for learning and teaching mathematics. Journal of mechanics of continua and mathematical sciences. 15. 1-12. https://doi.org/10.26782/jmcms.2020.04.00001

Arias-Rueda, J. H., Urdaneta, M. y Arias, M. (2018). Estrategias heurísticas en resolución de problemas a través de una experiencia integradora. Revista Sarance № 42, 43-64.

Arteaga-Martínez, B., Macías, J., \& Pizarro, N. (2020). Representation in the solution of mathematical problems: an analysis of metacognitive strategies of secondary education students. Uniciencia, 34(1), 263-280. https://doi.org/10.15359/ru.34-1.15

Ausubel D. P. (1965). Adquisición y retención del conocimiento: Una perspectiva cognitiva. Tercera edición. Barcelona. España: Paidós.

Boscán y Klever (2012). Metodología basada en el método heurístico de Poyla para el aprendizaje de la resolución de problemas matemáticos. Escenarios. 10(2), 7-19.

Brousseau, G. (1998). Theorie des situations didactiques. Grenoble, Francia: La Pensée Sauvage. 
Cuba Educa. (2015). Programa Heurístico General de Matemática. Portal Educativo Cubano. La habana Cuba.

Căprioară, D. (2015). Problem Solving - Purpose and Means of Learning Mathematics in School, Procedia. Social and Behavioral Sciences. Volume 191, pp. 1859-1864. ISSN 1877-0428. https://doi.org/10.1016/j.sbspro.2015.04.332.

Eisenmann P., Novotná J., Přibyl J., Břehovský, J., (2015). Developing a culture of problem solving with high school students through heuristic strategies. Math Ed Res J 27, 535-562 (2015). https://doi.org/10.1007/s13394-015-0150-2

Gardner H. (2016). Estructuras de la mente: La teoría de las inteligencias múltiples. Primera edición electrónica. México: Fondo de Cultura Económica.

Hernández, R., Fernández, C. y Baptista, P. (2010). Metodología de la Investigación. $5^{\text {ta }}$ Ed. México, D.F., México: McGraw Hill Interamericana.

Hitt F. y Quiroz R. S. (2017). Aprendizaje de las matemáticas a través de la modelación matemática en un medio sociocultural ligado a la teoría de la actividad. Revista Colombiana de Educación. Núm. 73. pp. 151-175.

Hoon T. S., Kee, K. L., Singh P., (2013). Learning mathematics using a heuristic approach. Revista ScienceDirect. Vol 90. 862-869. https://doi.org/10.1016/j.sbspro.2013.07.162

Lissabet, J. L. (2019). El enfoque axiológico del proceso de enseñanza-aprendizaje de la asignatura Matemática en la escuela primaria multigrada cubana. Revista dilemas contemporáneos. 7(1). 1-29. https://doi.org/10.46377/dilemas.v28i1.1604

Shrestha, I. M., Luitel, B. C., \& Pant, B. P. (2021). Exploring transformative pedagogy in teaching mathematics. Mathematics Education Forum Chitwan, Nepal.1-12

Medina (2017). Influencia del método heurístico para la enseñanza - aprendizaje de la matemática en alumnos del tercer grado de secundaria del distrito de Cajabamba. [Tesis doctoral, Universidad Privada Antenor Orrego]. https://repositorio.upao.edu.pe/handle/20.500.12759/2526

Ministerio de Educación, (2013) Rutas del Aprendizaje. Hacer uso de saberes matemáticos para afrontar desafíos diversos. Lima. Perú.

Ministerio de Educación (2016). Currículo nacional de educación. Lima. Perú.

Ministerio de Educación (2017). El Perú en Pisa 2015: Informe nacional de resultados. Primera edición. Lima. Perú: Oficina de Medición de la Calidad de los Aprendizajes. Recuperado de http://umc.minedu.gob.pe/wp-content/uploads/2017/04/Libro_PISA.pdf

Ministerio de Educación (2018). ¿Qué aprendizajes logran nuestros estudiantes? http://umc.minedu.gob.pe/wp-content/uploads/2018/10/Informe-Nacional-ECE-2018.pdf

Monereo C. (1998). Estrategias de enseñanza aprendizaje. Quinta edición. Barcelona. Editorial Graó.

Novotná, J., Eisenmann, P., Přibyl, J., Ondrušová, J., \& Břehovský, J. (2014). Problem solving in school mathematics based on heuristic strategies. Journal on Efficiency and Responsibility in Education and Science, 7(1), 1-6. https://doi.org/10.7160/eriesj.2014.070101

Neyra (2020). Aprendizaje Basado en Problemas para el Aprendizaje significativo en Matemática, en estudiantes de tercer año de secundaria, Chao 2019. [Tesis doctoral, Universidad César Vallejo]. https://hdl.handle.net/20.500.12692/44494

Peralta, J. (2000). Principios didácticos e históricos para la enseñanza de la matemática. España: Huerga Fierro.

Pólya, G. (1965). Cómo plantear y resolver problemas. México: Editorial Trillas. 
Pouyamanesh J., y Firoozeh L. (julio de 2013). The learning outcomes of students with mathematics in high and low tolerance for frustration were compared. Revista Ciberleninka. Recuperado de https://cyberleninka.org/article/n/258838

Pouyamanesh, J., \& Firoozeh, L. (2013). Compared the Learning Outcomes of Students with Math in High and Low Frustration Tolerance. Procedia - Social and Behavioral Sciences, Vol 84. 837-840. https://doi.org/10.1016/j.sbspro.2013.06.657

Pant, Binod \& Luitel, Bal \& Shrestha, Indra Mani. (2020). Incorporating STEAM Pedagogy in Teaching Mathematics. [Discurso principal]. Conference: Eighth International Conference to Review Research in Science, Technology and Mathematics EducationAt: Homi Bhabha Centre for Science Education, Mumbai, India.

Revelo Rosero, J. (2020). Impacto del uso de las TIC como herramientas para el aprendizaje de la matemática de los estudiantes de educación media. Cátedra, 1(1), 70-91. https://doi.org/10.29166/catedra.v1i1.764 (Original work published 26 de septiembre de 2018)

Sáenz Mass, E., Patiño Garcés, M., \& Robles González, J. (2018). Development of mathematical competences in geometric thinking, through Polya's heuristic method. Panorama, 11(21), 52-67. https://doi.org/10.15765/pnrm.v11i21.1055

Schoenfeld, A. (1985). Mathematical Problem Solving. Orlando: Academic Press. INC. Academic Press

Shakirova, K. B. Timerbayeva N. V.y Fazleeva E.I. (2018). Motivation in Teaching Mathematics. The European Proceedings of Social \& Behavioural Sciences. 206-215. https://doi.org/10.15405/epsbs.2018.09.24

Solis (2019). Estrategia heurística, trabajo colaborativo en el aprendizaje área de matemática de los estudiantes Red 6 UGEL. 2019. [Tesis doctoral, Universidad César Vallejo]. https://hdl.handle.net/20.500.12692/40187

Tambunan, H. (2018). Impact of Heuristic Strategy on Students' Mathematics Ability in High Order Thinking. International Electronic Journal of Mathematics Education, 13(3), 321328. https://doi.org/10.12973/iejme/3928

Tejeda (2017). Estrategias heurísticas y clima escolar en el aprendizaje de la matemática en estudiantes de primer año de secundaria de la Red 2 de la UGEL 03 - 2015. [Tesis doctoral, Universidad César Vallejo]. https://hdl.handle.net/20.500.12692/8450

Vygotsky, L. S. (1978). Mind in Society. Cambridge, MA: Harvard University Pres

Wang, K. (2012). Implications from Polya and Krutetskii. En: International Congress on Mathematical Education. 12., Seoul, Anais... Korea: COEX, 2012. 12 p.

\section{Anexos \\ Instrumentos de evaluación \\ Instrumento de Estrategias heurísticas}

Estimado estudiante contesta los siguientes ítems con la veracidad que corresponde para verificar la aplicabilidad de estrategias para tu aprendizaje en el área de matemática, tus respuestas son confidenciales para fines de investigación exclusivamente. 
Escala: Siempre (S), Casi siempre (CS), A veces (AV), Nunca (N).

\begin{tabular}{|l|c|c|c|c|}
\hline Escala: & Siempre (S) & Casi siempre (CS) & A veces (AV) & Nunca (N) \\
\hline & 4 & 3 & 2 & 1 \\
\hline
\end{tabular}

1. ¿Identificas con facilidad los datos que se presentan en un problema?
( ) Siempre
( ) Casi siempre
( ) A veces
( ) Nunca

2. ¿Sí existiera una o más incógnitas en un problema lo podrás identificar?
( ) Siempre
( ) Casi siempre
( ) A veces
( ) Nunca

3. ¿Luego de presentado el problema lo podrás explicar con tus propias palabras?
( ) Siempre
( ) Casi siempre
( ) A veces
( ) Nunca

4. ¿Para dar solución a un problema puedes identificar si los datos presentados son los suficientes?

( ) Siempre

( ) Casi siempre

( ) A veces

( ) Nunca

5. ¿Consideras que debes elegir un problema similar para resolver el propuesto?
( ) Siempre
( ) Casi siempre
( ) A veces
( ) Nunca

6. ¿Con los datos que te proporcionan en el problema puedes relacionarlos con algún material elegido?
( ) Siempre
( ) Casi siempre
( ) A veces
( ) Nunca

7. ¿Consideras realizar un dibujo, los cuales permitirán resolver el problema?
( ) Siempre
( ) Casi siempre
( ) A veces
( ) Nunca

8. ¿Es necesario elaborar algunas tablas para la solución de problemas?
( ) Siempre
( ) Casi siempre
( ) A veces 


\section{( ) Nunca}

9. ¿Utilizas una secuencialización de acuerdo a lo planificado anteladamente en la solución del problema?
( ) Siempre
( ) Casi siempre
( ) A veces
( ) Nunca

10. ¿Verificas si la solución del problema es la misma que has planificado al inicio?
( ) Siempre
( ) Casi siempre
( ) A veces
( ) Nunca

11. ¿Los gráficos que planteaste y las operaciones son favorables en la solución de los problemas?
( ) Siempre
( ) Casi siempre
( ) A veces
( ) Nunca

12. ¿El resultado al que llegaste es la solución para el problema que se ha planteado?
( ) Siempre
( ) Casi siempre
( ) A veces
( ) Nunca

13. ¿Al volver a leer el problema y examinas si los resultados que obtuviste responden a lo requerido?
( ) Siempre
( ) Casi siempre
( ) A veces
( ) Nunca

14. ¿Crees que es necesario realizar un análisis de la secuencialización de pasos utilizados para dar solución al problema verificando si fueron adecuados?
( ) Siempre
( ) Casi siempre
( ) A veces
( ) Nunca

15. ¿Te has propuesto volver a desarrollar el mismo problema, pero cambiando datos?

( ) Siempre

( ) Casi siempre

( ) A veces

( ) Nunca

16. ¿Consideras necesario aplicar otras estrategias diferentes a la tuyas, dadas a conocer por algún compañero?
( ) Siempre
( ) Casi siempre
( ) A veces 
( ) Nunca

\section{Prueba de matemática 5to. de secundaria}

\section{Competencia: $\quad$ Resuelve problemas de cantidad}

\section{Capacidades:}

Traduce cantidades a expresiones numéricas

Comunica su comprensión sobre los números y las operaciones

Usa estrategias y procedimientos de estimación y cálculo

Argumenta afirmaciones sobre las relaciones numéricas y las operaciones

Simboliza la proposición y determina su valor de verdad.

1. El arco iris tiene diez colores y solo tres de ellos son primarios
A) $\mathrm{p} v \mathrm{q}$, falso
B) $p^{\wedge} \mathrm{q}$, falso
C) $\mathrm{p}^{\wedge} \mathrm{q}$, verdadero
D) p v q, verdadero
E) p $\Delta \mathrm{q}$, falso

2. Se preguntó a 56 personas sobre su preferencia por tres marcas de zapatillas y se obtuvo la siguiente información:

30 prefieren las zapatillas de la marca A; 24 de la marca B; y 31 de la marca C.

10 personas prefieren las marcas A y B.

9 personas prefieren las marcas A y $\mathrm{C}$.

14 personas prefieren las marcas B y $\mathrm{C}$.

¿Cuántas personas prefieren las tres marcas de zapatilla?
A) 1
B) 2
C) 3
D) 4
E) 5

3. Una ameba se reproduce por bipartición, dividiéndose en dos cada hora. Si inicialmente hay 4 amebas, expresa en forma exponencial la cantidad de amebas que habrá al cabo de 8 horas.
A) $2^{6}$
B) $2^{7}$
C) $2^{8}$
D) $2^{9}$
E) $2^{10}$

4. Un albañil construye una fuente circular cuya circunferencia mide 58,4 m. ¿Cuánto mide aproximadamente el radio de la fuente?
A) 9,3
B) 9,6
C) 8,9
D) 9,1
E) 9,9

\section{Claves:}

B

D

E

A

\section{Competencia: $\quad$ Resuelve problemas de regularidad, equivalencia y cambio}

\section{Capacidades:}


Traduce datos y condiciones a expresiones algebraicas y gráficas

Comunica su comprensión sobre las relaciones algebraicas

Usa estrategias y procedimientos para encontrar equivalencias y reglas generales

Argumenta afirmaciones sobre relaciones de cambio y equivalencia.

5. La suma de los cuadrados de tres números naturales consecutivos es 590, hallar el mayor de los números.
A) 13
B) 14
C) 15
D) 16
E) 12

6. Una pieza rectangular tiene $8 \mathrm{~cm}$ más de largo que de ancho. Con esta se construye una caja de $900 \mathrm{~cm}^{3}$. Para ello, se cortará un cuadrado de $5 \mathrm{~cm}$ de lado en cada esquina y se doblarán sus bordes. Halla el largo de la caja.
A) $12 \mathrm{~cm}$
B) $13 \mathrm{~cm}$
C) $15 \mathrm{~cm}$
D) $18 \mathrm{~cm}$
E) $20 \mathrm{~cm}$

7. María Elena y Ramiro compraron una soga de 144m y cada uno lo dividió en varias partes iguales. Como cada pedazo de María Elena midió 8 metros menos que cada pedazo de Ramiro. María Elena obtuvo 3 pedazos más que este último, ¿En cuántos pedazos dividió María Elena la soga?
A) 9
B) 10
C) 11
D) 12
E) 13

8. Un carpintero hace cierto número de mesas. Vende 70 y le queda por vender más de la mitad. Después hace 6 mesas más y luego vende 36, y ahora le quedan menos de 42 por vender. ¿cuántas mesas hizo?
A) 141
B) 140
C) 135
D) $120 \mathrm{E}) 100$

\section{Claves}

$\mathrm{C}$

$\mathrm{D}$

A

A

\section{Competencia: $\quad$ Resuelve problemas de forma, movimiento y localización}

\section{Capacidades:}

Modela objetos en formas geométricas y sus transformaciones.

Comunica su comprensión sobre las formas y relaciones geométricas.

Usa estrategias y procedimientos para medir y orientarse en el espacio.

Argumenta afirmaciones sobre relaciones geométricas. 
9. Luis cerca un terreno de forma de un triángulo rectángulo si los lados $\mathrm{AB}$ y $\mathrm{BC}$ miden $6 \mathrm{~cm}$ y $8 \mathrm{~cm}$ respectivamente, hallar la medida del lado AC.

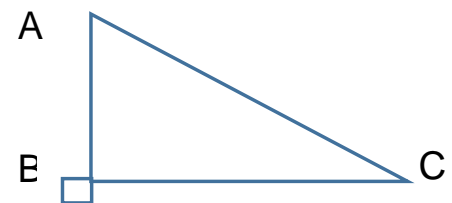
A) $5 \mathrm{~cm}$
B) $6 \mathrm{~cm}$
C) $7 \mathrm{~cm}$
D) $10 \mathrm{~cm}$
E) $12 \mathrm{~cm}$

10. Claudia camina por un parque de forma triangular y observa que dos de sus ángulos miden $30^{\circ}$ y $60^{\circ}$, respectivamente. ¿cuánto mide el tercer ángulo en radianes?
A) $\pi \mathrm{rad}$.
B) $\frac{\pi}{2} \mathrm{rad}$.
C) $2 \pi \mathrm{rad}$.
D) $3 \pi \mathrm{rad}$.
E) $\frac{2 \pi}{3} \mathrm{rad}$.

11. Una escalera de 10 metros de longitud está apoyada contra un muro de una construcción, de tal modo que forman un ángulo de $16^{0}$. Calcula la distancia aproximada del pie de la escalera al muro. (Considera sen $16^{0}=\frac{7}{25}$ )
A) $2,8 \mathrm{~m}$
B) $3,8 \mathrm{~m}$
C) $4,5 \mathrm{~m}$
D) $5,1 \mathrm{~m}$
E) $6,1 \mathrm{~m}$

12. A $180 \mathrm{~m}$ de la base de un edificio se observa la parte alta de este con un ángulo de elevación de $37^{0}$. Determine la altura del edificio.
A) $135 \mathrm{~m}$
B) $150 \mathrm{~m}$
C) $160 \mathrm{~m}$
D) $115 \mathrm{~m}$
E) $100 \mathrm{~m}$

\section{Claves}

D

B

A

A

\section{Competencia: $\quad$ Resuelve problemas de gestión de datos e incertidumbre}

\section{Capacidades:}

Representa datos con gráficos y medidas estadísticas o probabilísticas Comunica su comprensión de los conceptos estadísticos y probabilísticos.

Usa estrategias y procedimientos para recopilar y procesar datos.

Sustenta conclusiones o decisiones con base a la información obtenida.

13. El número de hermanos es una variable:
A) Cuantitativa continua
B) Cualitativa ordinal
C) Cualitativa nominal
D) Cuantitativa discreta
E) Cualitativa 
14. En una reunión de 100 estudiantes, la media aritmética de sus edades es 24. Si cada mujer tuviera 5 años más y cada hombre 4 años más, el nuevo promedio sería 28,6. ¿Cuántas mujeres hay en la reunión?
A) 40
B) 60
C) 64
D) 66
E) 80

15. Determina cuántos partidos de fútbol se deben programar en una rueda de un campeonato donde participan 16 equipos que jugarán todos contra todos.
A) 90
B) 100
C) 120
D) 150
E) 240

16. De los 78 participantes de un concurso de ecología, 45 son hombres. Si se elige un participante al azar, ¿cuál es la probabilidad de que sea mujer?
A) $2 / 3$
B) $11 / 26$
C) $12 / 26$
D) $5 / 26$
E) $33 / 26$

Claves:
D
B
C
B

\section{Ficha técnica de Estrategias heurísticas}

1. Nombre del instrumento: Cuestionario de Estrategias heurísticas

2. Autores: Mg. Víctor Hugo Medina Pérez; Dr. Manuel Ángel Pérez Azahuanche

3. Objetivo: Medir el nivel de utilización de las Estrategias heurísticas utilizados por estudiantes de secundaria de la Institución Educativa № 81014, Trujillo, 2020.

4. Usuarios: Alumnos del $5^{\circ}$ grado de educación secundaria de la Institución Educativa $\mathrm{N}^{\circ}$ 81014, Trujillo 2020 .

5. Tiempo: 45 minutos

\section{Procedimientos de aplicación:}

Se compartirá un link con los estudiantes para que puedan acceder a contestar el cuestionario de Estrategias heurísticas

Se administrará por única vez el instrumento de Estrategias heurísticas

Las instrucciones están presentes en el cuestionario siendo leídas por los estudiantes.

El tiempo de desarrollo del cuestionario Estrategias heurísticas será de 45 minutos.

El cuestionario resuelto será registrado de manera automática a medida que respondan los estudiantes. 


\section{Organización de ítems:}

\begin{tabular}{|c|c|c|}
\hline DIMENSIÓN & INDICADORES & ÍTEMS \\
\hline $\begin{array}{l}\text { Comprensión } \\
\text { del enunciado }\end{array}$ & $\begin{array}{l}\text { Identifican los datos de los problemas. } \\
\text { Identifican las condiciones de los problemas. } \\
\text { Identifican la incógnita presente en el problema a resolver. } \\
\text { Identifican si los datos existentes son suficientes para } \\
\text { ejecutar el proceso. }\end{array}$ & $1,2,3,4$ \\
\hline $\begin{array}{l}\text { Concepción } \\
\text { de un plan }\end{array}$ & $\begin{array}{l}\text { Buscan estrategias que ayuden a la solución del problema. } \\
\text { Utilizan herramientas que ayuden a resolver cada } \\
\text { problema planteado. } \\
\text { Elaboran gráficos representando la solución al problema a } \\
\text { resolver. } \\
\text { Explican el plan pertinente que ayuda a la solución del } \\
\text { problema. }\end{array}$ & $5,6,7,8$ \\
\hline $\begin{array}{l}\text { Ejecución del } \\
\text { plan }\end{array}$ & $\begin{array}{l}\text { Aplican cada una de las estrategias que ha hecho. } \\
\text { Utilizan alguna estructura matemática para la solución del } \\
\text { problema. } \\
\text { Utilizan operaciones simbólicas que ayuden a la } \\
\text { resolución de los problemas. }\end{array}$ & $9,10,11,12$ \\
\hline $\begin{array}{l}\text { Visión } \\
\text { retrospectiva }\end{array}$ & $\begin{array}{l}\text { Comprueban y comparan la solución al problema con } \\
\text { otros que le sirvan de apoyo. } \\
\text { Repiensan sobre las estrategias utilizadas. } \\
\text { Evalúan otras formas para la solución de los problemas. } \\
\text { Cambian los datos del problema y los contextos } \\
\text { propuestos para resolver otros problemas. }\end{array}$ & $13,14,15,16$ \\
\hline
\end{tabular}

\section{Escala:}

a. Escala general:

\begin{tabular}{cc}
\hline Escala & Rangos \\
\hline Logro destacado & De 49 a 64 \\
Logro esperado & De 33 a 48 \\
Proceso & De 16 a 32 \\
Inicio & De 1 a 15 \\
\hline
\end{tabular}

\section{b. Escala específica:}

\begin{tabular}{ccccc}
\hline \multirow{2}{*}{ Nivel } & \multicolumn{4}{c}{ Dimensiones } \\
\cline { 2 - 5 } & $\begin{array}{c}\text { Comprensión del } \\
\text { enunciado. }\end{array}$ & $\begin{array}{c}\text { Concepción de } \\
\text { un plan. }\end{array}$ & $\begin{array}{c}\text { Ejecución del } \\
\text { plan. }\end{array}$ & $\begin{array}{c}\text { Visión } \\
\text { retrospectiva. }\end{array}$ \\
\hline $\begin{array}{c}\text { Logro } \\
\text { destacado }\end{array}$ & {$[13-16]$} & {$[13-16]$} & {$[13-16]$} & {$[13-16]$}
\end{tabular}




$\begin{array}{ccccc}\begin{array}{c}\text { Logro } \\ \text { esperado }\end{array} & {[09-12[} & {[09-12[} & {[09-12[} & {[09-12[} \\ \text { Proceso } & {[05-08[} & {[05-08[} & {[05-08[} & {[05-08[} \\ \text { Inicio } & {[01-04[} & {[01-04[} & {[01-04[} & {[01-04[}\end{array}$

\section{Ficha técnica del Aprendizaje de la matemática}

1. Nombre del instrumento: Prueba objetiva del Aprendizaje de la Matemática.

2. Autores: Mg. Víctor Hugo Medina Pérez; Dr. Manuel Ángel Pérez Azahuanche.

3. Objetivo: Medir el nivel de Aprendizaje de la matemática alcanzado por estudiantes de secundaria de la Institución Educativa $\mathrm{N}^{\circ}$ 81014, Trujillo 2020.

4. Usuarios: Alumnos del $5^{\circ}$ grado de educación secundaria de la Institución Educativa $\mathrm{N}^{\circ}$ 81014, Trujillo 2020.

5. Tiempo: 90 minutos

6. Procedimientos de aplicación:

Se compartirá un link con los estudiantes para que puedan acceder a contestar la prueba objetiva del Aprendizaje de la matemática

Se administrará por única vez el instrumento de Aprendizaje de la matemática

Las instrucciones están presentes en la prueba objetiva siendo leídas por los estudiantes.

El tiempo de desarrollo de la prueba objetiva del Aprendizaje de la matemática será de 90 minutos.

La prueba objetiva resuelta será registrada de manera automática a medida que respondan los estudiantes.

\section{Organización de ítems:}

\begin{tabular}{|c|c|c|}
\hline DIMENSIÓN & INDICADORES & ÍTEMS \\
\hline Resuelve & Convertir la cantidad a una terminología numérica & $1,2,3,4$ \\
\hline $\begin{array}{l}\text { Problemas de } \\
\text { cantidad }\end{array}$ & $\begin{array}{l}\text { Comunique su conocimiento de los números y las operaciones. } \\
\text { Discutir declaraciones sobre operaciones y relaciones } \\
\text { numéricas. } \\
\text { Gestionar habilidades e instrucciones de apreciación y cálculo. }\end{array}$ & \\
\hline $\begin{array}{l}\text { Resuelve } \\
\text { Problemas de } \\
\text { regularidad, } \\
\text { equivalencia y } \\
\text { cambio }\end{array}$ & $\begin{array}{l}\text { Convierta datos y condiciones en representación algebraica y } \\
\text { gráfica } \\
\text { Participa en la razón de las relaciones algebraicas } \\
\text { Utiliza habilidades y ordenamientos para hallar equivalencia y } \\
\text { reglas generales. } \\
\text { Dar a conocer argumentos sobre intercambio v reciprocidad. }\end{array}$ & $5,6,7,8$ \\
\hline $\begin{array}{l}\text { Resuelve } \\
\text { Problemas de } \\
\text { forma, }\end{array}$ & $\begin{array}{l}\text { Modelar cosas con formas geométricas y sus innovaciones. } \\
\text { Comunique su razón de formas geométricas y relaciones. } \\
\text { Emplear destrezas y ordenamientos para medir la zona y } \\
\text { determinar la dirección del espacio. }\end{array}$ & $9,10,11,12$ \\
\hline
\end{tabular}


movimiento y Discutir declaraciones sobre condiciones geométricas.

localización

\begin{tabular}{lll}
$\begin{array}{l}\text { Resuelve } \\
\text { Problemas de } \\
\text { gestión de }\end{array}$ & Presentar datos con figuras y estadísticas o medidas probables & $13,14,15,16$ \\
datos e & probabilidad. \\
incertidumbre & Utilizar tácticas y procesos para coleccionar y procesar datos. \\
& $\begin{array}{l}\text { Respaldar desenlaces o disposiciones basadas en la indagación } \\
\text { obtenida. }\end{array}$ \\
\hline
\end{tabular}

\section{Escala:}

a. Escala general:

\begin{tabular}{ll}
\hline Escala & Rangos \\
\hline Logro destacado & De 49 a 64 \\
Logro esperado & De 33 a 48 \\
Proceso & De 16 a 32 \\
Inicio & De 01 a 15 \\
\hline
\end{tabular}

b. Escala específica:

\begin{tabular}{ccccc}
\hline NIVEL & $\begin{array}{c}\text { Resuelve } \\
\text { Problemas de } \\
\text { cantidad }\end{array}$ & $\begin{array}{c}\text { Resuelve Problemas de } \\
\text { regularidad, } \\
\text { equivalencia y cambio }\end{array}$ & $\begin{array}{c}\text { Resuelve Problemas } \\
\text { de forma, } \\
\text { movimiento y } \\
\text { localización }\end{array}$ & $\begin{array}{c}\text { Resuelve } \\
\text { Problemas de } \\
\text { gestión de datos } \\
\text { e incertidumbre }\end{array}$ \\
\hline $\begin{array}{c}\text { Logro } \\
\text { destacado } \\
\text { Logro }\end{array}$ & {$[13-16]$} & {$[13-16]$} & {$[13-16]$} & {$[13-16]$} \\
$\begin{array}{c}\text { esperado } \\
\text { Proceso }\end{array}$ & {$[05-12[$} & {$[09-12[$} & {$[09-12[$} & {$[09-12[$} \\
Inicio & {$[01-04[$} & {$[05-08[$} & {$[05-08[$} & {$[05-08[$} \\
\hline
\end{tabular}

